\title{
The effects of gamelike features and test location on cognitive test performance and participant enjoyment
}

\author{
Jim Lumsden ${ }^{\text {Corresp., }}$ 1,2 , Andy Skinner ${ }^{1,2}$, Andy T Woods ${ }^{2}$, Natalia S Lawrence ${ }^{3}$, Marcus Munafò ${ }^{1,2}$ \\ 1 MRC Integrative Epidemiology Unit (IEU), University of Bristol, Bristol, United Kingdom \\ 2 School of Experimental Psychology, University of Bristol, Bristol, United Kingdom \\ 3 School of Psychology, College of Life and Environmental Sciences, University of Exeter, Exeter, United Kingdom \\ Corresponding Author: Jim Lumsden \\ Email address: jim.lumsden@bristol.ac.uk
}

Computerised cognitive assessments are a vital tool in the behavioural sciences, but participants often view them as effortful and unengaging. One potential solution is to add gamelike elements to these tasks in order to make them more intrinsically enjoyable, and some researchers have posited that a more engaging task might produce higher quality data. This assumption, however, remains largely untested.

We investigated the effects of gamelike features and test location on the data and enjoyment ratings from a simple cognitive task. We tested three gamified variants of the Go-No-Go task, delivered both in the laboratory and online. In the first version of the task participants were rewarded with points for performing optimally. The second version of the task was framed as a cowboy shootout. The third version was a standard Go-No-Go task, used as a control condition. We compared reaction time, accuracy and subjective measures of enjoyment and engagement between task variants and study location.

We found points to be a highly suitable game mechanic for gamified cognitive testing because they did not disrupt the validity of the data collected but increased participant enjoyment. However, we found no evidence that gamelike features could increase engagement to the point where participant performance improved. We also found that while participants enjoyed the cowboy themed task, the difficulty of categorising the gamelike stimuli adversely affected participant performance, increasing No-Go error rates by $28 \%$ compared to the non-game control. Responses collected online vs. in the laboratory had slightly longer reaction times but were otherwise very similar, supporting other findings that online crowdsourcing is an acceptable method of data collection for this type of research. 


\section{The effects of gamelike features and test location on cognitive test performance and participant enjoyment}

Jim Lumsden ${ }^{1,2}$, Andy Skinner ${ }^{1,2}$, Andy Woods $^{2}$, Natalia Lawrence ${ }^{3}$, Marcus Munafò ${ }^{1,2}$

${ }^{1}$ MRC Integrative Epidemiology Unit (IEU), University of Bristol, Bristol, United Kingdom

${ }^{2}$ School of Experimental Psychology, University of Bristol, Bristol, United Kingdom

${ }^{3}$ School of Psychology,

College of Life and Environmental Sciences, University of Exeter, Exeter, United Kingdom

* Corresponding author

E-mail: jim.lumsden@bristol.ac.uk

Address: Jim Lumsden, 12a Priory Rd, School of Experimental Psychology, University of Bristol, BS8 1TU, UK 


\begin{abstract}
Computerised cognitive assessments are a vital tool in the behavioural sciences, but participants often view them as effortful and unengaging. One potential solution is to add gamelike elements to these tasks in order to make them more intrinsically enjoyable, and some researchers have posited that a more engaging task might produce higher quality data. This assumption, however, remains largely untested.

We investigated the effects of gamelike features and test location on the data and enjoyment ratings from a simple cognitive task. We tested three gamified variants of the Go-No-Go task, delivered both in the laboratory and online. In the first version of the task participants were rewarded with points for performing optimally. The second version of the task was framed as a cowboy shootout. The third version was a standard Go-No-Go task, used as a control condition. We compared reaction time, accuracy and subjective measures of enjoyment and engagement between task variants and study location.

We found points to be a highly suitable game mechanic for gamified cognitive testing because they did not disrupt the validity of the data collected but increased participant enjoyment. However, we found no evidence that gamelike features could increase engagement to the point where participant performance improved. We also found that while participants enjoyed the cowboy themed task, the difficulty of categorising the gamelike stimuli adversely affected participant performance, increasing No-Go error rates by $28 \%$ compared to the non-game control. Responses collected online vs. in the laboratory had slightly longer reaction times but were otherwise very similar, supporting other findings that online crowdsourcing is an acceptable method of data collection for this type of research.
\end{abstract}




\section{The effects of gamelike features and test location on data and participant enjoyment in cognitive testing}

\section{Introduction}

Cognitive tasks are a common tool in psychological research, but are often viewed as effortful and unengaging by participants (D’Angiulli \& LeBeau, 2002; Healy, Kole, Buck-Gengler, \& Bourne, 2004). If participants are bored by an assessment, then their lack of motivation may have negative effects on data quality, adding noise and leading to suboptimal performance (DeRight \& Jorgensen, 2014). One potential solution is to add gamelike elements to these tasks in order to make them more intrinsically enjoyable (e.g. McPherson \& Burns, 2008; Prins, Ponsioen, van der Oord, \& Dovis, 2011). By using game mechanics which incentivise maximal performance, participants' goals might be adjusted from 'completing the experiment as quickly as possible' to 'trying to succeed at the game', thus producing better data (Hawkins, Rae, Nesbitt, \& Brown, 2013). For example, a cognitive task with a points mechanic might incentivise rapid responding and consistently high accuracy by awarding points in relation to these measures..

However, the assumption that a more engaging task will provide better data is largely untested. Some studies have reported a positive effect of game mechanics on participant performance (Dovis, Oord, Wiers, \& Prins, 2011; Ninaus et al., 2015), although most have shown mixed results (e.g. Katz, Jaeggi, Buschkuehl, Stegman, \& Shah (2014); see Lumsden, Edwards, Lawrence, Coyle, \& Munafo, (In review) for a review). There is some evidence that gamelike tests do not improve performance, and may in fact worsen it (Hawkins et al., 2013), potentially by introducing new task demands. In contrast, many studies have shown that gamelike experiments are more motivating and less boring (Dörrenbächer, Müller, Tröger, \& Kray, 2014; Lumsden, Attwood, \& Munafo, In review; McPherson \& Burns, 2007; Tong \& Chignell, 2014).

Miranda \& Palmer (2014) investigated the effects of two different game mechanics, sounds and points. They found that sound effects slowed reaction times, but points did not, showing that some game elements may have more impact on task performance than others. Further systematic research is required to understand how specific gamelike features impact the quality of data gathered from, and participant ratings of, cognitive tasks.

Another recent development in cognitive research is the deployment of cognitive tasks on online platforms. One of the key enablers of online research is Mechanical Turk (MTurk; www.mturk.com), an Amazon-based 'work marketplace' which allows users to sign up, complete small online tasks and receive reimbursement for their time. While MTurk is often used for non-research purposes, it has grown popular in the behavioural sciences because it enables the testing of large numbers of people in a very short time. However, studies investigating the comparability of data from laboratory and online testing versions of tasks have again reported mixed findings (Crump, McDonnell, \& Gureckis, 2013; Schreiner, Reiss, \& Schweizer, 2014). These differences may arise from a number of factors, including: differences in the population sampled (with online participants tending to be older than those recruited through traditional methods), differences in hardware used to run a given study, the suitability of the remote environment for concentration and reduced motivation due to lack of experimenter presence (see Woods, Velasco, Levitan, Wan, \& Spence, 2015). Interestingly, Hawkins et al., (2013) also found that participants' self-reported enjoyment and engagement was much lower when the task was online, but it was unclear why this was the case. 
In this study we aimed to investigate the effects of gamelike features and the effects of test location on the data collected by and participant enjoyment of a simple cognitive task. We used three variations of the Go-No-Go task (GNG), delivered both in the laboratory and online using MTurk: one variant where participants were rewarded with points for performing optimally, one where the task was framed as a cowboy shootout (game theme), and a standard GNG task as a control condition. Both the laboratory and online arms of the study used Xperiment, a web-based platform for psychological experiments (www.xperiment.mobi), as a delivery method.

\section{Methods}

\section{Design and Overview}

The aim of this study was to compare three versions of GNG-task, each with different gamelike features (non-game, points, theme) across two different testing sites (laboratory and online). We used a between-subjects design, with reaction times (RT) on Go trials, Go trial accuracy, No-Go trial accuracy and subjective ratings as the dependent variables of interest. We did not expect to see a difference in median RTs or mean No-Go accuracy between any of the GNG variations. We had no expectations regarding differences in Go accuracy between the task variants. Finally, based on effects found previously, we did anticipate that participants would rate both the theme variant and points variant favourably compared to the non-game control. We also expected all ratings to be lower on average in the online.

\section{Participants}

Participants who were tested in the laboratory were staff and students recruited through existing email lists and poster advertisements around the University of Bristol. They received either course credit or $£ 3$ in compensation for their time. Participants who were tested online signed up to the study through MTurk; they received payment of $\$ 1.50$. We required that participants were older than 18 years of age, did not have a diagnosis of ADHD and were not colour blind.

Once enrolled, participants were randomly assigned to one of the three task variants. Since testing site (laboratory or online) was determined by the participant's method of signup, the groups were not matched. The laboratory condition included 28 participants in each task variant and the online condition included roughly 72 participants in each task variant. Precise allocation of equal numbers of participants to each task variant could not be achieved online due to multiple concurrent signups to the experiment-platform.

\section{Materials \\ Online and Laboratory platforms}

In order to eliminate task differences caused by variations in delivery platform, we used Xperiment to host both the lab and the online version of the task. Xperiment is an online experimental platform which has been shown to collect comparable data to other, offline, test software (Knoeferle, Woods, Käppler, \& Spence, 2015; Michel, Woods, Neuhäuser, Landgraf, \& Spence, 2015). Laboratory participants were seated in a computer cubical while they completed the task and the questionnaire via the internet. They used a PC with a mouse and keyboard to complete the task. MTurk participants accessed exactly the same experimental software, but via their own PC or laptop. 
116

117

118

119

120

121

122

123

124

125

126

127

128

129

130

131

132

133

134

135

136

137

138

139

140

141

142

143

144

145

146

147

148

149

150

151

152

153

154

155

156

157

158

159

160

\section{Go/No-Go Task}

The Go/No-Go task (GNG) is a measure of response inhibition (the ability to stop or withhold a motor response), which is a key feature of executive control (Verbruggen \& Logan, 2008). The main cognitive tasks that are used to assess response inhibition include: the stopsignal task, which imposes a delay between a stimulus and a stop signal, thus placing demands on 'action cancellation' (inhibition of an initiated response); and the GNG task, which is a qualitatively different 'action restraint' task (Schachar et al., 2007). It comprises a reaction-time task with a set of fixed no-action stimuli. It measures inhibitory control by repeatedly presenting stimuli to which the participant must respond rapidly, while occasionally presenting stimuli to which the participant must avoid responding.

We developed our own GNG task for use on the Xperiment platform, based on the tasks used by Benikos, Johnstone, \& Roodenrys, (2013) and Bowley et al., (2013), but with custom features for each variant. Each trial began with a fixation cross displayed in the middle of the screen, $500 \mathrm{~ms}$ later a picture appeared in the centre of the screen and remained for $600 \mathrm{~ms}$. On Go trials the participant had to respond to the stimuli as fast as they could by pressing the spacebar within this $600 \mathrm{~ms}$ window. In No-Go trials (signalled by the image content) they simply had to withhold their response. Each trial was followed by a variable inter-trial-interval (ITI) of 500-1000 ms. If the participant responded incorrectly, the ITI was replaced by a feedback screen, failed No-Go trials resulted in a red cross overlaid on the stimuli, while incorrect no-responses were followed by "Too slow" written in red text.

The task consisted of 5 blocks of 60 trials each. Between each block a pause screen was displayed and the participant had to wait for 10 seconds. Each block contained 5 sub-blocks of 12 trials, and each sub-block consisted of 9 Go trials and 3 No-Go trials, in randomised order. In total, the task contained 75 No-Go trials $(25 \%)$ and 225 Go trials $(75 \%)$ and took around 11 minutes to complete. GNG tasks vary widely in their design, but using $25 \%$ No-Go trials is similar to several other studies (Benikos et al., 2013; Kertzman et al., 2008; Watson \& Garvey, 2013; Yechiam et al., 2006).

Non-Game Variant: The non-game control used a stimulus set consisting of a diverse range of 20 everyday objects: 15 green and 5 red. Go trials used the green object, and No-Go trials used the red objects (see Figure 1). We selected green and red objects to ensure that the non-game variant was as intuitive as the themed variant, as these colours are commonly associated with going and stopping (Moller, Elliot, \& Maier, 2009). See Supplementary Figure 1 for the instructions presented to the participants.

Points Variant: The points variant was identical in structure to the non-game control, except that a scoring system was added, based on that used in Miranda \& Palmer, (2014). The participant's score was displayed in middle of the screen, to the left of the stimuli (see Figure 1). On each successful Go trial the participant earned points equal to bonus*(600-RT)/10. This bonus was a multiplier $(2 \mathrm{x}, 4 \mathrm{x}, 8 \mathrm{x} \ldots$...) which doubled every 5 trials but was reset to $\mathrm{x} 1$ when the participant made a No-Go error. On a successful inhibition the bonus was not lost, but no points were awarded. This reward/punishment scheme also fits with findings of Guitart-Masip et al., (2012), who found that subjects were much more successful in learning active choices when rewarded for them, and passive choices when punished. The points awarded in the previous trial 
161 were displayed in the centre of the screen during the ITI. The instructions framed the task as a 162 game, see Supplementary Figure 2.

163

164

165

166

167

168

169

170

171

172

173

174
Theme Variant: The theme variant also used the same format as non-game control, except with the addition of a theme designed to provide a narrative framework for the action required by the task (see Supplementary Figure 3). The participant was introduced to the task as a shooting game, where they were the sheriff of a small town and a group of criminals had holed up in a saloon and taken hostages. The GNG task proceeded as above but the stimuli were replaced with cartoon characters; with cowboys as Go targets and innocent civilians as No-Go targets (see Figure 1). Throughout each block a cartoon saloon graphic remained on the screen, with stimuli appearing in the doorway. When the participant pressed the response key a blood splat was overlaid onto the current stimuli for the remainder of the trial time. Feedback was presented in the ITI, as in the non-game control. The stimulus set consists of 15 cowboys and 5 innocent civilians. 


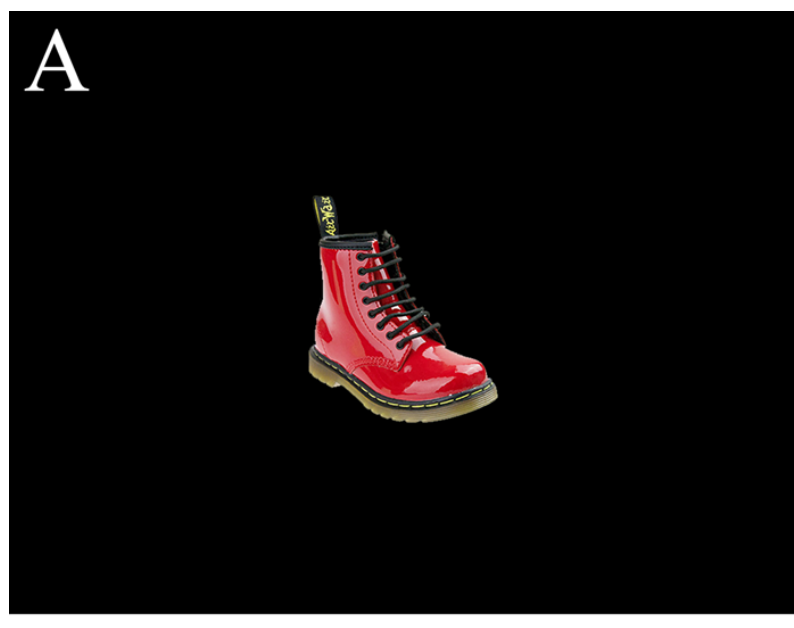

\section{B}
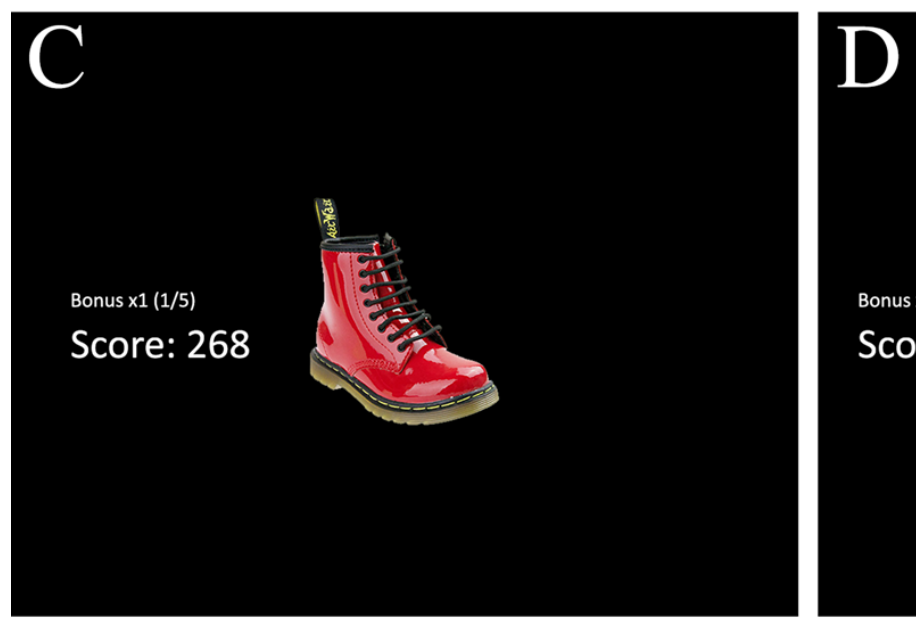

Bonus $\times 16(4 / 5)$

Score: 3570

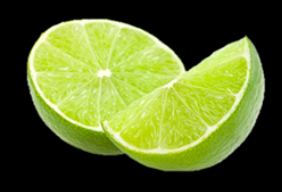

\section{E}
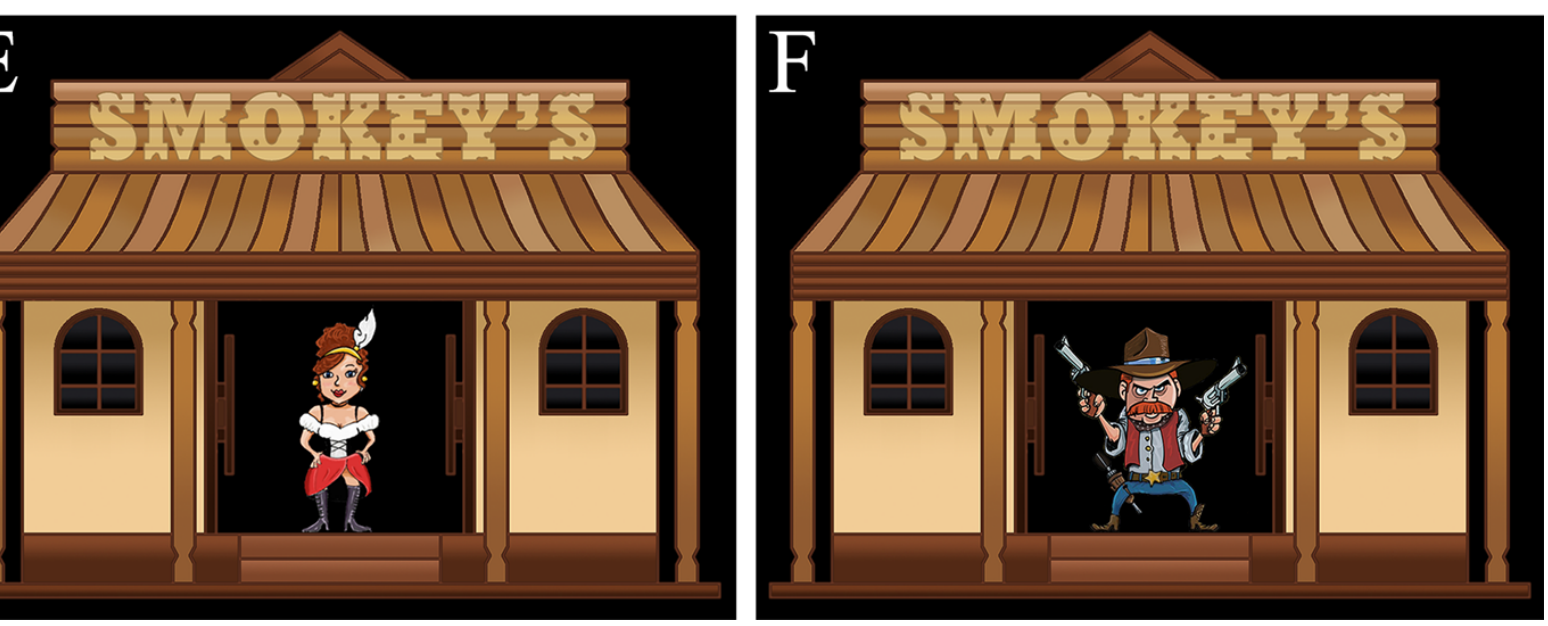

Figure.1: A: No-Go trial from the control variant. B: Go trial from the control variant. C: No-Go trial from the points variant. D: Go trial from the points variant. E: No-Go trial from the theme variant. F: Go trial from the theme variant.

\section{Assessment of Enjoyment and Engagement}

After completing the task participants were given a short questionnaire to assess their opinion of the task. Following assessment approaches by Hawkins et al (2013) and Miranda \& Palmer (2014), 11 questions were selected : 1) How enjoyable did you find the task? 2) How 
183

frustrating did you find the task? 3) Was it difficult to concentrate for the duration of the task? 4) How well do you think you performed on this task? 5) How mentally stimulating did you find this task to be? 6) How boring did you find the task? 7) How much effort did you put in throughout the task? 8) How repetitive was the task? 9) How willing would you be to take part in the study again? 10) How willing would you be recommend the study to a friend? 11) How intuitive did you find the pictures chosen for stop and for Go? Participants responded using a continuous visual analogue scale (VAS), presented as a horizontal line with a label at either end and no subdivisions. Participants marked a point between these two labels using their mouse.. The questionnaire was delivered using the same Xperiment platform that delivered the tasks.

\section{Procedure}

Study sessions lasted approximately 15 minutes. Each participant took part in only one task variant in order to minimise the duration of the study and prevent fatigue. Participants confirmed that they met the inclusion criteria and provided consent using an online form. We then collected demographic information on the participant's age, sex, ethnicity, level of education and the number of hours they spent playing video games per week. Instructions for the task were then displayed The GNG task was then delivered, followed by the questionnaire and finally a debrief screen was displayed. Participants were free to withdraw from the study at any point by simply closing the browser window, this would result in no data being saved. The study was pre-registered on the Open Science Framework (https://osf.io/va547/) and ethical approval was obtained from the Faculty of Science Research Ethics Committee at the University of Bristol (22421). The study was conducted according to the revised Declaration of Helsinki (2013).

\section{Statistical Analysis}

Since we did not anticipate a difference in RTs or mean No-Go accuracy between task variants, we initially decided not to use a Frequentist approach as it is not ideal for testing equivalences (Berger \& Sellke, 1987; Blackwelder, 1982): Bayesian analyses are better suited to this (Wetzels, Raaijmakers, Jakab, \& Wagenmakers, 2009). However, upon collection and initial exploration of the data it was apparent that large differences did exist and so we updated our statistical plan to include both Frequentist and Bayesian approaches.

\section{Sample size determination}

At the time of study design, no previous study had investigated differences in data produced by gamelike and non-gamelike GNG tasks, and therefore we had no previous effect size on which to base a sample size determination. We selected a sample size for the laboratory condition to provide sufficiently dense distributions to allow for meaningful analysis. For the online condition we scaled up our sample-size to take advantage of the larger samples possible with crowdsourcing.

\section{Reaction time data}

Reaction time data were summarised by median Go RTs for each participant. Differences between task variants and testing sites were assessed using box-plots and two-way ANOVAs. Where Frequentist approaches found no evidence of a difference between two means, we used Bayesian t-tests to assess the evidence for equality (Rouder, Speckman, Sun, Morey, \& Iverson, 2009). A Bayesian t-test produces a Bayes Factor, which either provides evidence to support one of two hypotheses, or implies the data are insensitive, see Table.1. In our analysis one hypothesis 
was always "the mean difference is zero" and the other was "the mean difference is not zero". We used the Bayesian t-test procedure from the R-Package BayesFactor (http://bayesfactorpcl.rforge.r-project.org/), with a naïve JZS prior.

\begin{tabular}{|l|l|l|}
\hline $\begin{array}{l}\text { Hypothesis 0: } \\
\text { The difference between } \\
\text { means is } 0\end{array}$ & Strength of Evidence & $\begin{array}{l}\text { Hypothesis 1: } \\
\text { The difference between } \\
\text { means is between } 0-\mathrm{X}\end{array}$ \\
\hline $.33 \leq \mathrm{BF} \leq 1$ & No support either way & $1 \leq \mathrm{BF} \leq 3$ \\
\hline $.1 \leq \mathrm{BF} \leq .33$ & Positive & $3 \leq \mathrm{BF} \leq 10$ \\
\hline $.01 \leq \mathrm{BF} \leq .1$ & Strong & $10 \leq \mathrm{BF} \leq 100$ \\
\hline $\mathrm{BF}<.01$ & Decisive & $\mathrm{BF}>100$ \\
\hline
\end{tabular}

Table.1: Interpreting Bayes factors (adapted from Raftery,1995)

\section{Accuracy data}

Accuracy data were handled similarly. We calculated \% accuracy scores on Go and NoGo trials for each participant. Differences between task variants and sites were assessed using box-plots and two-way ANOVAs. Where we found no evidence of a difference between two means, we used Bayesian t-tests to weigh the evidence for equality.

\section{Questionnaire data}

We assessed differences in participant ratings both visually and using a two-way ANOVA of total score with site and task variant as factors. Total score was computed by averaging the VAS scores from items 1-10 (with items 2,3,6 and 8 reversed) to produce a score out of 100 .

\section{Results}

\section{Characteristics of Participants}

A total of 304 participants took part in this study, however four participants from the online group were excluded from subsequent analyses because we did not record any responses from them for the duration of the GNG task. A further thirteen participants were excluded from the analysis due to extremely poor Go accuracy rates (more than 4 inter-quartiles ranges away from the median).

Excluding outliers, 287 participants took part: 84 in the laboratory (mean age $=21, \mathrm{SD}=$ $4,26 \%$ male) and 203 online (mean age $=35, \mathrm{SD}=11,50 \%$ male). A chi-square test indicated that the number of male participants in the laboratory site was statistically different to the online $\left(X^{2}(1, \mathrm{~N}=287)=14.012, p<.001\right)$. A t-test provided evidence for difference in ages between the laboratory group and online $(t(285)=16.35, p<.001)$, with the online participants typically being older. Participants who took part online reported slightly more hours spent playing computer games per week (median $=$ " 1 to $5 ")$ than those that took part in the lab (median = " 0 ") - there was evidence that the distributions of responses for both groups differed, with the laboratory group being skewed towards 0 (Mann-Whitney $U=3994$, Online $=203$, Lab $=84, p<$ .001 two-tailed). Online participants also reported higher levels of education (median $=$ "Bachelor's degree") than those in the laboratory (median = "High School Graduate"), and there was evidence that these distributions differed, with $83 \%$ of the laboratory group being high school graduates and the online group being a relatively even split between high school 
268

graduates and university graduates (Mann-Whitney $U=5330$, Online $=203$, Lab $=84, p<.001$ ). However, given that the majority of laboratory participants were undergraduates, they will be more than equally educated within a few years. Ethnicity also differed between sites $\left(X^{2}(4, \mathrm{~N}=\right.$ $287)=20.456, p<.001)$ : both groups featuring a high proportion of participants of European ancestry (69\% in the laboratory, $85 \%$ online,) but we saw a higher proportion of East Asian participants in the laboratory sample (14\% vs 4\%). Screen resolution in the laboratory was 1920 x 1080, median screen resolution online was 1440 x 900 .

\section{Go Trial Data}

Data from Go trials in all three variants and on both sites are shown in Figures 2 and 3 and Supplementary Tables 1 and 2. A two-way ANOVA of the median Go RTs indicated main effects of both task variant $\left(\mathrm{F}[2,281]=174.891, p<.001, \eta^{2}=.56\right)$, and site $(\mathrm{F}[1,281]=$ $\left.24.906, p<.001, \eta^{2}=.08\right)$; however, there was no evidence of an interaction $(p=.298)$. Go RTs were longer online and were also longest in the theme variant. Post-hoc t-tests showed RTs from the theme variant to be longer than the points $(\mathrm{t}(190)=16.316, p<.001, d=2.37)$ and non-game $(\mathrm{t}(186)=16.991, p<.001, d=2.49)$ variants; however, we could not detect a difference between the points and non-game variants $(\mathrm{t}(192)=.085, p=.932, d=.01)$. We therefore compared the non-game and points variants using a Bayesian t-test and found good evidence that Go RTs were equal in the non-game and points variants (Bayes factor $=0.157$ ). We also performed exploratory analysis into the effect of task duration on RT, see the Supplementary Analyses document.

Accuracy followed a similar pattern. A two-way ANOVA found evidence for main effects of both task variant $\left(\mathrm{F}[2,281]=72.974, p<.001, \eta^{2}=.34\right)$ and site $(\mathrm{F}[1,281]=15.277$, $\left.p<.001, \eta^{2}=.05\right)$. Again, there was no clear evidence of an interaction $(p=.143)$. Go accuracy was generally very high, as expected. However, it was slightly lower online, see Figure 3. Posthoc t-tests showed that the theme variant had lower accuracy than the points $(t(104.1)=10.347$, $p<.001, d=2.03)$ and non-game $(t(115.8)=9.413, p<.001, d=1.75)$ variants. We could not detect a difference between the points and non-game variants $(\mathrm{t}(170.9)=1.511, p=.133, d=$ .23 ) and a Bayesian t-test to compare the points and non-game variants for equality suggested there was insufficient evidence to support either equality or a difference (Bayes factor $=0.459$ ). Due to the non-normality of the data, we also used Mann-Whitney U tests to confirm the ANOVA findings, see Supplementary Table 3. 


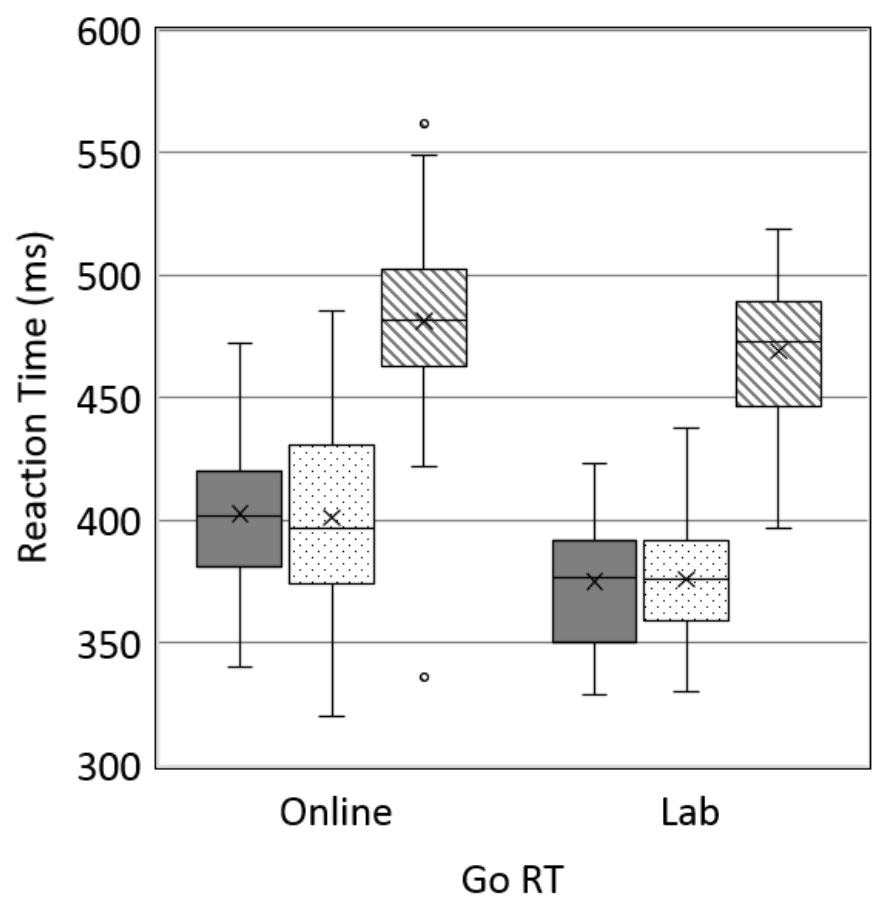

$\square$ Non-game $\square$ Points $\nabla$ Theme

Figure.2: Box and Whisker plots of Median Go RTs, split by task variant and site.

\section{No-Go Trial Data}

Data from No-Go trials in all three variants and from both sites are shown in Figure 3 and Supplementary Tables 1 and 2. A two-way ANOVA of No-Go accuracy data found evidence of a main effect of task variant $\left(\mathrm{F}[2,281]=247.362, p<.001, \eta^{2}=.64\right)$, but no evidence for an effect of site or an interaction ( $p \mathrm{~s}>.393)$. No-Go accuracy was much lower in the theme variant than the other two variants, and post-hoc t-tests showed that the theme variant was different to the points $(t(106.5)=18.396, p<.001, d=3.57)$ and non-game $(t(114.7)=17.582, p<.001, d=$ $3.28)$ variants. Again, we could not detect a difference between the points and non-game variants $(t(180.9)=1.012, p=.313, d=0.15)$ but a Bayesian t-test found good evidence that No-Go accuracy was equivalent in the non-game and points variants (Bayes factor $=0.253$ ). Additionally, we performed exploratory analysis into the effect of task duration on No-Go accuracy, see the Supplementary Analyses document.

We saw ceiling effects in both the points and non-game variants, which resulted in skewed distributions. Due to the non-normality of the data, we used Mann-Whitney U tests to check the results of the post-hoc t-tests of Go and No-Go Accuracy between task variants, see Supplementary Table 3. All Mann-Whitney U tests confirmed the findings of the t-tests. 


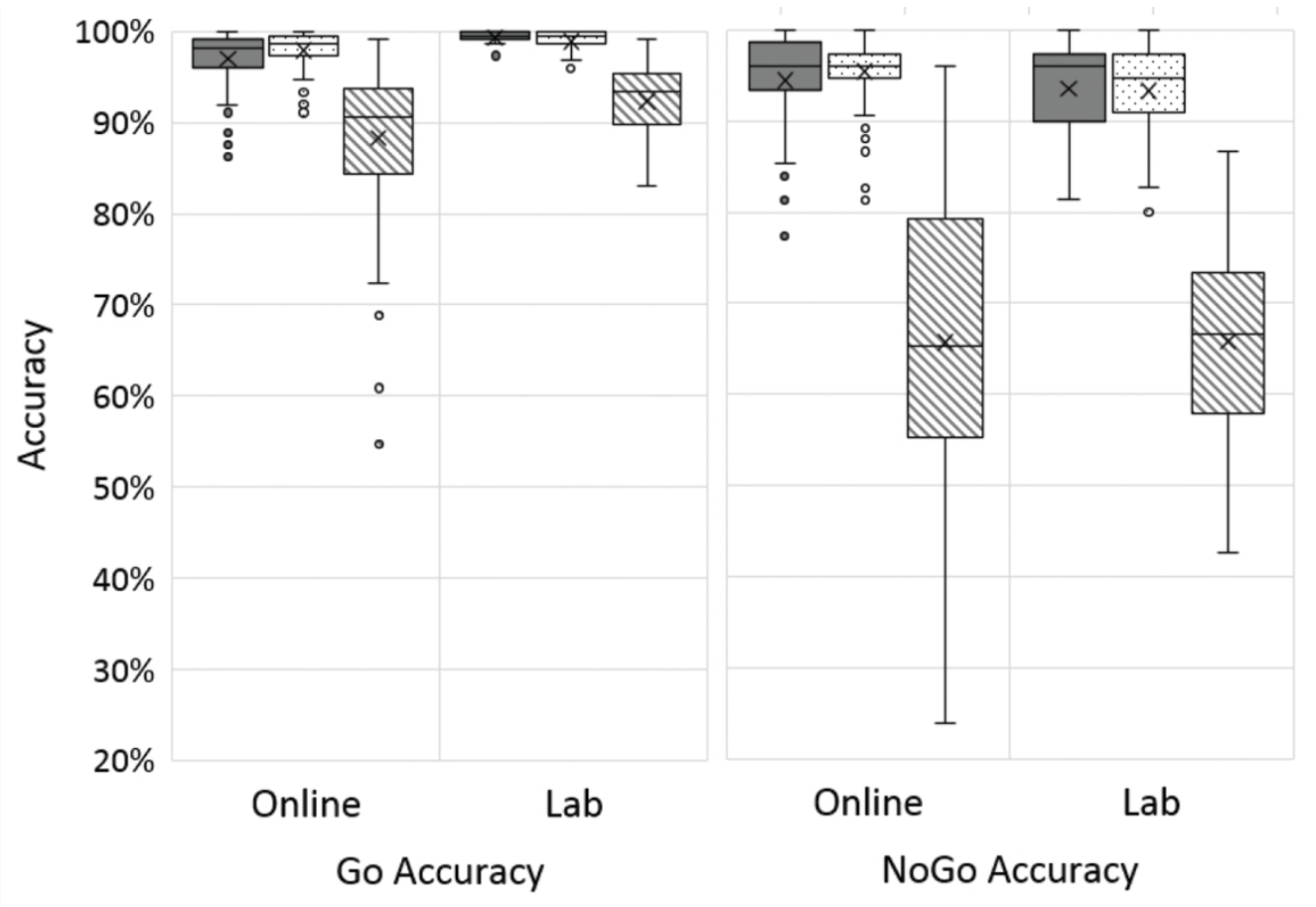

$\square$ Non-game $\square$ Points $\triangle$ Theme

Figure.3: Box and Whisker plots of Go and No-Go Accuracy, split by task variant and site

\section{Subjective Questionnaire of Enjoyment and Engagement}

Supplementary Table 4 shows the mean VAS scores from the engagement questionnaire, by site and task variant. In general, subjective engagement scores were slightly higher online $(\mathrm{t}(285)=$ $2.732, p<.001, d=0.32$ ). Online participants rated all the task variants as more repetitive than those in the laboratory group, yet were much more willing to take part in the study again.

A two-way ANOVA of total-score data found evidence of a main effect of task variant ( $\mathrm{F}$ $\left.[2,281]=3.613, p=.028, \eta^{2}=.03\right)$ and site $\left(\mathrm{F}[1,281]=7.423, p=.007, \eta^{2}=.03\right)$. Post-hoc ttests showed that the non-game variant was rated lower than the points variant $(t(192)=2.986, p$ $=.003, d=0.43)$, but no other differences were found $(p \mathrm{~s}>.178)$.

We suspected that the heterogeneity in group composition might be driving the difference in total score between the laboratory group and the online group. To assess we performed a twoway ANCOVA of total-score data with site and task variant as factors, and age and sex as covariates. Again we found evidence of a main effect of task variant $(\mathrm{F}[2,279]=3.070, p=$ $\left..048, \eta^{2}=.02\right)$, but not for site, sex, age or an interaction ( $\left.p \mathrm{~s}>.084\right)$. This implies that the difference in scores between the two sites was indeed an artefact of age/sex preferences, and that task variant was the primary factor driving a difference in scores, see Figure 4. 


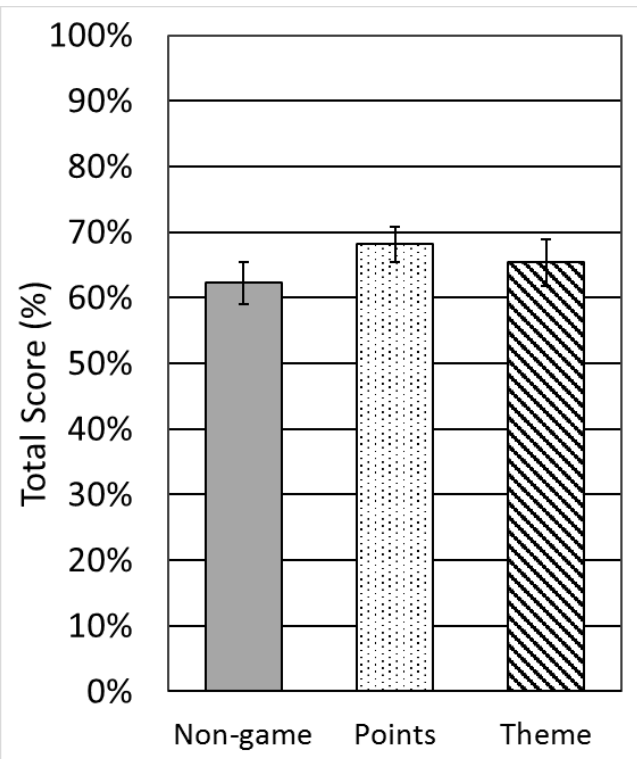

Figure.4: Mean total scores from the subjective questionnaire, split by variant and adjusted for age and sex.

We performed two Bayesian t-tests to investigate whether the total scores of either the points and theme variants or the non-game and theme variants could be considered equal, but found that the data supported neither equality or inequality (Bayes factors $=0.322$ and 0.372 respectively).

Figure 5 shows the individual item questionnaire scores broken down by task variant alone. The pattern found in the total scores is apparent in the individual questionnaire items also, with the points variant being rated slightly more favourably. The non-game control was clearly rated as the least enjoyable and stimulating, the most boring and the most frustrating. Participants also reported putting less effort into this variant than others. The theme variant had mixed scores, with participants feeling they performed poorly and finding it very frustrating; however, it does appear that the cowboy stimuli resulted in the task being less repetitive, and on several measures, such as enjoyment, it does not differ from the points variant. Overall, the points variant was best received: these participants were the most willing to recommend the study to a friend, as well as willing to put the most effort into the task. We found no difference between any of the three variants on ratings of "difficulty concentrating" or "intuitive pictures". 


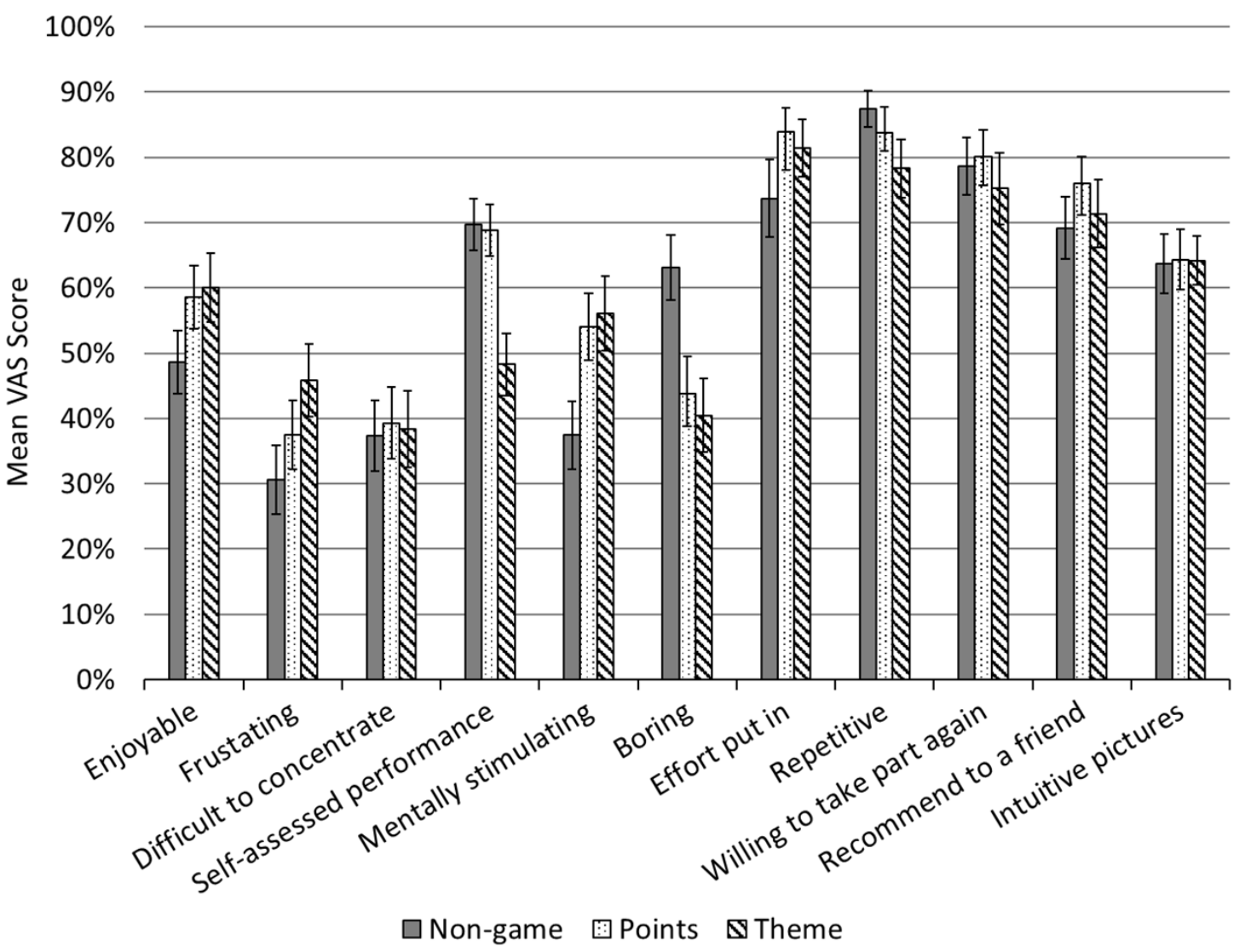

Figure.5: VAS scores for individual questions on the subjective engagement questionnaire, split by task variant.

\section{Discussion}

\section{Comparison of Task Site (Online vs Laboratory)}

The laboratory group was a fairly unrepresentative sample consisting mainly of young, female undergraduates who volunteered for the experiment. The MTurk group had a much more balanced demographic, with a range of ages, education levels and games experience. Although MTurk users are also a self-selected group, their slightly wider demographic lends some ecological validity to our findings. We also saw some differences between the online and laboratory group in terms of the behavioural data we collected. RTs recorded from MTurk users were $\sim 25 \mathrm{~ms}$ longer on average. Notably, we saw no difference in No-Go accuracy, implying that online participants were just as able to inhibit their responses to stimuli.

There are several possible reasons for the longer RTs online. Lower participant effort may have played a role, potentially resulting from the absence of an experimenter in the room, environmental distractions or a difference in perceived reimbursement value. However, there is some evidence that MTurk participants can have higher levels of attentiveness and performance than laboratory participants (Hauser \& Schwarz, 2015). One might expect age to play a role in longer RTs, yet we saw no correlation between age and median Go RT. The fact that we saw no increase in No-Go accuracy as a result of the longer RTs, as would be expected, suggests that the difference is artificial and due to technical reasons. Although we used the same experimental platform to test both groups, there are still several potential sources of slowing such as differing 
386

387

388

389

390

391

392

393

394

395

396

397

398

399

400

401

402

403

404

405

406

407

408

409

410

411

412

413

414

415

416

417

418

419

420

421

422

423

424

425

426

427

428

429

430

431

operating systems, keyboards and web browsers (Neath, Earle, Hallett, \& Surprenant, 2011; Plant \& Turner, 2009; Woods et al., 2015).

Despite the difference in RTs, we saw no interactions between site and task variant, and there were no unusual patterns of performance between the two groups. Our results show that online cognitive testing can produce valid and useful data, as long as one is aware of potentially longer RTs.

The online group generally rated all the task variants more highly on the subjective questionnaire. This stands in direct contrast to Hawkins et al. (2013) who reported lower engagement scores when the task was delivered online. Adjusting for age and sex eliminated the difference between the sites, implying that it was not the difference of testing location that influenced enjoyment, but rather the difference in sample composition. The theme variant was rated particularly highly online, and this may be due to the greater levels of gaming experience in the online group.

\section{Comparing Task Variants}

It is clear from our results that the theme variant was much more difficult than the other two variants, with longer RTs and lower accuracy rates. We propose several possible reasons for this: increased difficulty of spotting stimuli against the background image, a reluctance to shoot people (even cartoon characters) and the complexity of the stimuli. There was likely too much overlap in colour and pose between the civilians and cowboys, resulting in a slower categorisation of Go and No-Go stimuli.

Our motivation for using red and green objects as opposed to simple stop and go symbols was to match the intuitiveness of stop and go stimuli across task variants (i.e., we felt that shooting the cowboys and avoiding the innocents would be so intuitive that the non-game condition would need equally intuitive stimuli). However, the association between red/green and stop/go may have been stronger than we expected (see Moller et al., 2009) and there is evidence that attending to colour is easier than attending to shape (McDermott, Pérez-Edgar, \& Fox, 2007). These factors may have made the points and non-game variants easier than anticipated, although any implicit association between red/stop and green/go may have been unnoticed by participants as they reported that stimuli in the theme variant were equally intuitive to those in the points and non-game variants.

The clear differences between the theme and non-game variants invalidates the use of these stimuli for gathering data comparable to non-gamelike GNG tasks. This represents an important finding since several previous studies have used complex stimuli, such as robots and monsters, in their gamified cognitive tasks (Dörrenbächer et al., 2014; Dovis et al., 2011; Prins et al., 2011). The idea of using graphics alone to gamify a task is not uncommon, but future researchers must ensure that the addition of gamelike stimuli does not make their task considerably more difficult. Detrimental effects on participant performance resulting from the introduction of gamelike features have been found before (Katz et al., 2014; Miranda \& Palmer, 2014), and it is likely that complicating a task too much may increase its difficulty such that the data it collects becomes incomparable to data from a traditional task.

Boendermaker, Boffo, \& Wiers, (2015) investigated the use of gamelike features in a GNG alcohol-bias training task, and although they saw no overall effect of the training, they also found no evidence of a difference in training efficacy between the gamelike and non-gamelike variants. Their gamelike variant was themed and contained points, lives and levels, so their results stand in contrast to our more minimal theme variant which had a negative impact on 
432 participant performance. This inconsistency is likely because Boendermaker's task clearly 433 delineated the stimuli from the themed surroundings of the game, i.e. using extrinsic fantasy

434

435

436

437

438

439

440

441

442

443

444

445

446

447

448

449

450

451

452

453

454

455

456

457

458

459

460

461

462

463

464

465

466

467

468

469

470

471

472

473

474

475

476

477
(Malone, 1981), rather than actually gamifying the stimuli as we did.

When we consider the data collected by the non-game and points variants, the Bayesian analyses we performed provide good evidence that these tasks produced equivalent data. Our points system was not particularly punishing and this may explain why we saw no impact of the points system on behaviour. There is evidence that a GNG task which rewards participants for fast responding and punishes them for failed inhibitions can optimise performance (GuitartMasip et al., 2012), but our study did not detect any improvement in data as a result of the points mechanic. Nevertheless, the points variant received the highest total score both online and in the lab, implying that points are a highly enjoyable game mechanic. This is interesting because adding points to cognitive tasks in order to make them more engaging is not uncommon, but to the best of our knowledge this is the first study to directly compare the appeal of points against another game mechanic.

Finally, it is clear from our results that the addition of even a single gamelike feature makes a huge difference to the participants' perception of the task. The non-game control was rated as far more boring, far less enjoyable and less mentally stimulating than either of the task variants. Although our results show this theme to be of secondary appeal to points, this may be inaccurate given that the theme variant was more difficult. As such, it comes as no surprise that participants rated it as more frustrating and felt they performed less well. Future work might investigate the role of theme more effectively by carefully controlling task difficulty. We also highlight the need for replication of our findings, with points being compared against other themes or in other contexts, such as longitudinal studies.

\section{Limitations and Conclusions}

We consider the difference in difficulty between the theme variant and the other task variants to be the most important limitation of this study. This difference is informative because gamelike stimuli and complex visual environments are common in gamified tasks, and our results highlight the need to limit the impact of these features. Although, clearly, such variations in accuracy mean we are limited in the manner in which we can compare task performance across variants. Secondly, we opted for a between-subjects design which does not allow us to study the impact of different gamelike features on an individual's performance, confounds hardware/individual differences with effects caused by the task variant. Nevertheless, the large sample size we achieved using online testing helps to counteract the lack of power associated with our experimental design. We also acknowledge that our design is not suitable to validate our task for the measurement of response inhibition, and that we would require a within-subjects design in order to test predictive validity (see also Kato, (2013) and Boendermaker, Prins, \& Wiers, (2015)). Thirdly, the task we used was quite short in duration, meaning that participants may not have had time to become bored enough to affect the data, even when playing the nongame variant. If participants were not that bored by the task, then this may have limited the potential effects of gamification. Future research might explore whether longer task durations result in greater boredom, and therefore greater impact of gamification. Finally, although we intended the questionnaire to measure enjoyment and engagement, the fact that it is delivered after the task means that the scores likely represent only a post-hoc appraisal of enjoyment of the task. In future work we intend to use a more objective measure of engagement, such as a dual task paradigm, to test for differences between the variants. 
478

479

480

481

482

483

484

485

486

487

488

489

490

491

492

In conclusion, we found points to be a highly suitable game mechanic for gamified cognitive testing, in that they do not disrupt the validity of the data collected and yet increase participant enjoyment. This will be welcome news to experimenters who need to increase participant engagement. For example, several recent studies have used GNG tasks to train automatic inhibition to specific stimuli, such as food or alcohol (Jones et al., 2014; Lawrence et al., 2015; Veling, van Koningsbruggen, Aarts, \& Stroebe, 2014). Such studies require participants to complete several sessions of GNG training and therefore rely on high levels of engagement; our data suggest that simply adding points may achieve this goal.

Despite some hope that gamelike features might increase engagement to the point where participant performance improves, we found no evidence of such an effect in this study. We also found that while participants enjoyed the themed task and its visually interesting stimuli, the complexity of categorising such stimuli can adversely affect participant performance. Finally, we found differences in the data collected online and in the lab, with slightly longer participant RTs online, but we saw no interactions or unusual patterns. This suggests that online crowdsourcing is a very acceptable method of data collection for this type of research. 


\section{References}

Benikos, N., Johnstone, S. J., \& Roodenrys, S. J. (2013). Varying task difficulty in the Go/Nogo task: The effects of inhibitory control, arousal, and perceived effort on ERP components. International Journal of Psychophysiology, 87(3), 262-272. http://doi.org/10.1016/j.ijpsycho.2012.08.005

Berger, J. O., \& Sellke, T. (1987). Testing a Point Null Hypothesis: The Irreconcilability of P Values and Evidence. Journal of the American Statistical Association, 82(397), 112-122. http://doi.org/10.1080/01621459.1987.10478397

Blackwelder, W. C. (1982). "Proving the null hypothesis" in clinical trials. Controlled Clinical Trials, 3(4), 345-353. http://doi.org/10.1016/0197-2456(82)90024-1

Boendermaker, W. J., Boffo, M., \& Wiers, R. W. (2015). Exploring Elements of Fun to Motivate Youth to Do Cognitive Bias Modification. Games for Health Journal, 4(6), 434-443. http://doi.org/10.1089/g4h.2015.0053

Boendermaker, W. J., Prins, P. J. M., \& Wiers, R. W. (2015). Cognitive Bias Modification for adolescents with substance use problems - Can serious games help? Journal of Behavior Therapy and Experimental Psychiatry, 49, Part A, 13-20. http://doi.org/10.1016/j.jbtep.2015.03.008

Bowley, C., Faricy, C., Hegarty, B., J. Johnstone, S., L. Smith, J., J. Kelly, P., \& A. Rushby, J. (2013). The effects of inhibitory control training on alcohol consumption, implicit alcohol-related cognitions and brain electrical activity. International Journal of Psychophysiology, 89(3), 342-348. http://doi.org/10.1016/j.ijpsycho.2013.04.011

Crump, M. J. C., McDonnell, J. V., \& Gureckis, T. M. (2013). Evaluating Amazon's Mechanical Turk as a Tool for Experimental Behavioral Research. PLoS ONE, 8(3), e57410. http://doi.org/10.1371/journal.pone.0057410

D'Angiulli, A., \& LeBeau, L. S. (2002). On Boredom and Experimentation in Humans. Ethics \& Behavior, 12(2), 167-176. http://doi.org/10.1207/S15327019EB1202_4

DeRight, J., \& Jorgensen, R. S. (2014). I Just Want My Research Credit: Frequency of Suboptimal Effort in a Non-Clinical Healthy Undergraduate Sample. The Clinical Neuropsychologist, 0, 1-17. http://doi.org/10.1080/13854046.2014.989267

Dörrenbächer, S., Müller, P. M., Tröger, J., \& Kray, J. (2014). Dissociable effects of game elements on motivation and cognition in a task-switching training in middle childhood. Frontiers in Psychology, 5, 1275. http://doi.org/10.3389/fpsyg.2014.01275

Dovis, Oord, Wiers, \& Prins. (2011). Can Motivation Normalize Working Memory and Task Persistence in Children with Attention-Deficit/Hyperactivity Disorder? The Effects of Money and Computer-Gaming. Journal of Abnormal Child Psychology, 40(5), 669-681. http://doi.org/10.1007/s10802-011-9601-8

Guitart-Masip, M., Huys, Q. J. M., Fuentemilla, L., Dayan, P., Duzel, E., \& Dolan, R. J. (2012). Go and no-go learning in reward and punishment: interactions between affect and effect. NeuroImage, 62(1), 154-166. http://doi.org/10.1016/j.neuroimage.2012.04.024

Hauser, D. J., \& Schwarz, N. (2015). Attentive Turkers: MTurk participants perform better on online attention checks than do subject pool participants. Behavior Research Methods, 18. http://doi.org/10.3758/s13428-015-0578-z

Hawkins, G. E., Rae, B., Nesbitt, K. V., \& Brown, S. D. (2013). Gamelike features might not improve data. Behavior Research Methods, 45(2), 301-318. http://doi.org/10.3758/s13428-012-0264-3 
539

540

541

542

543

544

545

546

547

548

549

550

551

552

553

554

555

556

557

558

559

560

561

562

563

564

565

566

567

568

569

570

571

572

573

574

575

576

577

578

579

580

581

582

583

584
Healy, A. F., Kole, J. A., Buck-Gengler, C. J., \& Bourne, L. E. (2004). Effects of Prolonged Work on Data Entry Speed and Accuracy. Journal of Experimental Psychology: Applied, 10(3), 188-199. http://doi.org/10.1037/1076-898X.10.3.188

Jones, A., McGrath, E., Houben, K., Nederkoorn, C., Robinson, E., \& Field, M. (2014). A comparison of three types of web-based inhibition training for the reduction of alcohol consumption in problem drinkers: study protocol. BMC Public Health, 14, 796. http://doi.org/10.1186/1471-2458-14-796

Kato, P. M. (2013, April 25). What do you mean when you say your serious game has been validated? Experimental vs. Test Validity. Retrieved from http://www.webcitation.org/6gt9POLIu

Katz, B., Jaeggi, S., Buschkuehl, M., Stegman, A., \& Shah, P. (2014). Differential effect of motivational features on training improvements in school-based cognitive training. Frontiers in Human Neuroscience, 8. http://doi.org/10.3389/fnhum.2014.00242

Kertzman, S., Lowengrub, K., Aizer, A., Vainder, M., Kotler, M., \& Dannon, P. N. (2008). Gono-go performance in pathological gamblers. Psychiatry Research, 161(1), 1-10. http://doi.org/10.1016/j.psychres.2007.06.026

Knoeferle, K. M., Woods, A., Käppler, F., \& Spence, C. (2015). That Sounds Sweet: Using Cross-Modal Correspondences to Communicate Gustatory Attributes. Psychology \& Marketing, 32(1), 107-120. http://doi.org/10.1002/mar.20766

Lawrence, N. S., O’Sullivan, J., Parslow, D., Javaid, M., Adams, R. C., Chambers, C. D., ... Verbruggen, F. (2015). Training response inhibition to food is associated with weight loss and reduced energy intake. Appetite, 95, 17-28. http://doi.org/10.1016/j.appet.2015.06.009

Lumsden, Attwood, \& Munafo. (In review). Comparison of Data Generated by Gamified and Standard Stop Signal Tasks. Nature Scientific Reports.

Lumsden, J., Edwards, E., Lawrence, N., Coyle, D., \& Munafo, M. (In review). Gamification of cognitive assessment and cognitive training: A systematic review of applications and efficacy.

Malone, T. W. (1981). What Makes Things Fun to Learn? A Study of Intrinsically Motivating Computer Games. Pipeline, 6(2), 50.

McDermott, J. M., Pérez-Edgar, K., \& Fox, N. A. (2007). Variations of the flanker paradigm: Assessing selective attention in young children. Behavior Research Methods, 39(1), 6270. http://doi.org/10.3758/BF03192844

McPherson, \& Burns. (2007). Gs Invaders: Assessing a computer game-like test of processing speed. Behavior Research Methods.

McPherson, J., \& Burns, N. R. (2008). Assessing the validity of computer-game-like tests of processing speed and working memory. Behavior Research Methods, 40(4), 969-981. http://doi.org/10.3758/BRM.40.4.969

Michel, C., Woods, A. T., Neuhäuser, M., Landgraf, A., \& Spence, C. (2015). Rotating plates: Online study demonstrates the importance of orientation in the plating of food. Food Quality and Preference, 44, 194-202. http://doi.org/10.1016/j.foodqual.2015.04.015

Miranda, A. T., \& Palmer, E. M. (2014). Intrinsic motivation and attentional capture from gamelike features in a visual search task. Behavior Research Methods, 46(1), 159-172. http://doi.org/10.3758/s13428-013-0357-7

Moller, A. C., Elliot, A. J., \& Maier, M. A. (2009). Basic hue-meaning associations. Emotion (Washington, D.C.), 9(6), 898-902. http://doi.org/10.1037/a0017811 
585

586

587

588

589

590

591

592

593

594

595

596

597

598

599

600

601

602

603

604

605

606

607

608

609

610

611

612

613

614

615

616

617

618

619

620

621

622

623

624

625

626

627

628

629

630

Neath, I., Earle, A., Hallett, D., \& Surprenant, A. M. (2011). Response time accuracy in Apple Macintosh computers. Behavior Research Methods, 43(2), 353-362. http://doi.org/10.3758/s13428-011-0069-9

Ninaus, M., Pereira, G., Stefitz, R., Prada, R., Paiva, A., Neuper, C., \& Wood, G. (2015). Game elements improve performance in a working memory training task. International Journal of Serious Games, 2(1). http://doi.org/10.17083/ijsg.v2i1.60

Plant, R. R., \& Turner, G. (2009). Millisecond precision psychological research in a world of commodity computers: New hardware, new problems? Behavior Research Methods, 41(3), 598-614. http://doi.org/10.3758/BRM.41.3.598

Prins, Ponsioen, van der Oord, \& Dovis. (2011). Does computerized working memory training with game elements enhance motivation and training efficacy in children with ADHD? Cyberpsychology, Behavior and Social Networking.

Rouder, J. N., Speckman, P. L., Sun, D., Morey, R. D., \& Iverson, G. (2009). Bayesian t tests for accepting and rejecting the null hypothesis. Psychonomic Bulletin \& Review, 16(2), 225237. http://doi.org/10.3758/PBR.16.2.225

Schachar, R., Logan, G. D., Robaey, P., Chen, S., Ickowicz, A., \& Barr, C. (2007). Restraint and Cancellation: Multiple Inhibition Deficits in Attention Deficit Hyperactivity Disorder. Journal of Abnormal Child Psychology, 35(2), 229-238. http://doi.org/10.1007/s10802006-9075-2

Schreiner, M., Reiss, S., \& Schweizer, K. (2014). Method Effects on Assessing Equivalence of Online and Offline Administration of a Cognitive Measure: The Exchange Test. International Journal of Internet Science, 9(1), 52-63.

Tong, T., \& Chignell, M. (2014). Developing a Serious Game for Cognitive Assessment: Choosing Settings and Measuring Performance. In Proceedings of the Second International Symposium of Chinese CHI (pp. 70-79). http://doi.org/10.1145/2592235.2592246

Veling, H., van Koningsbruggen, G. M., Aarts, H., \& Stroebe, W. (2014). Targeting impulsive processes of eating behavior via the internet. Effects on body weight. Appetite, 78, 102109. http://doi.org/10.1016/j.appet.2014.03.014

Verbruggen, F., \& Logan, G. D. (2008). Automatic and controlled response inhibition: Associative learning in the go/no-go and stop-signal paradigms. Journal of Experimental Psychology: General, 137(4), 649-672. http://doi.org/10.1037/a0013170

Watson, T. D., \& Garvey, K. T. (2013). Neurocognitive correlates of processing food-related stimuli in a Go/No-go paradigm. Appetite, 71, 40-47. http://doi.org/10.1016/j.appet.2013.07.007

Wetzels, R., Raaijmakers, J. G. W., Jakab, E., \& Wagenmakers, E.-J. (2009). How to quantify support for and against the null hypothesis: A flexible WinBUGS implementation of a default Bayesian t test. Psychonomic Bulletin \& Review, 16(4), 752-760. http://doi.org/10.3758/PBR.16.4.752

Woods, A. T., Velasco, C., Levitan, C. A., Wan, X., \& Spence, C. (2015). Conducting perception research over the internet: a tutorial review. PeerJ, 3, e1058. http://doi.org/10.7717/peerj.1058

Yechiam, E., Goodnight, J., Bates, J. E., Busemeyer, J. R., Dodge, K. A., Pettit, G. S., \& Newman, J. P. (2006). A Formal Cognitive Model of the Go/No-Go Discrimination Task: Evaluation and Implications. Psychological Assessment, 18(3), 239-249. 